\title{
ACESSÓRIOS DE IMOBILIZAÇÃO E PROTEÇÃO UTILIZADOS NA RADIOTERAPIA DE PEQUENOS ANIMAIS
}

\author{
Michel Campos Vettorato ${ }^{1}$ \\ Marco Antonio Rodrigues Fernandes ${ }^{2}$ \\ Luiz Carlos Vulcano ${ }^{3}$
}

\begin{abstract}
RESUMO
A radioterapia é uma modalidade médica bastante estabelecida no tratamento do câncer dentro da medicina veterinária mundialmente. Os protocolos radioterápicos aplicados em animais variam em função de vários fatores. Na maioria dos procedimentos requer a sedação ou mesmo a anestesia do animal e este fato impõe o uso de acessórios de imobilização especialmente desenvolvidos para as diferentes espécies de animais tratados e conforme os respectivos procedimentos indicados. Por isso este trabalho tem como objetivo descrever os tipos de acessórios utilizados para imobilização e para a proteção de pequenos animais submetidos à radioterapia. Para o seu desenvolvimento uma pesquisa bibliográfica foi realizada por meio de sites de buscas como Google Acadêmico, Scielo, Bireme, PubMed, além de consultas em livros da biblioteca do campus da UNESP de Botucatu. Apesar das limitações de cada acessório avaliado nessa revisão, foi possível identificar a utilização de cada um e como isso pode ser vantajoso para o tratamento dos animais submetidos a radioterapia.
\end{abstract}

Palavras-chave: radioterapia veterinária, pequenos animais, acessórios de imobilização.

\section{ACCESSORIES FOR DETENTION AND PROTECTION USED IN SMALL ANIMALS RADIATION THERAPY}

\begin{abstract}
Radiation therapy is a medical method well established in the treatment of cancer in veterinary medicine worldwide. The radiotherapy protocols applied in animals vary according to several factors. In most procedures require sedation or anesthesia of the animal and this fact imposes the use of immobilization accessories specially developed for the different species of animals and treated as their specified procedures. Therefore, this study aims to describe the types of accessories used for immobilization and for the protection of small animals undergoing radiotherapy. For its development a literature search was performed by search sites like Google Scholar, Scielo, Bireme, PubMed, and consultations in books campus library Botucatu UNESP. Despite the limitations of each accessory rated this review, it was possible to identify the use of each and how this can be advantageous for the treatment of animals undergoing radiation therapy.
\end{abstract}

Keywords: veterinary radiotherapy, little animals, Immobilization accessories.

\footnotetext{
${ }^{1}$ Mestrando em Biotecnologia Animal na FMVZ-UNESP-Botucatu. Contato para correspondência.

${ }^{2}$ Docente do Departamento de Dermatologia e Radioterapia da Faculdade de Medicina, UNESP-Botucatu.

${ }^{3}$ Docente da Faculdade de Medicina Veterinária e Zootecnia de Botucatu - UNESP.
} 


\title{
ACCESORIOS INMOVILIZACIÓN Y PROTECCIÓN UTILIZADOS EN RADIOTERAPIA DE PEQUEÑOS ANIMALES
}

\begin{abstract}
RESUMEN
La radioterapia es un método médico bien establecida en el tratamiento del cáncer en la medicina veterinaria en todo el mundo. Los protocolos de radioterapia aplicada en los animales varían de acuerdo a varios factores. En la mayoría de los procedimientos requieren sedación o anestesia del animal y este hecho impone el uso de accesorios de inmovilización especialmente desarrollado para las diferentes especies de animales y tratados como sus procedimientos especificados. Por lo tanto, este estudio tiene como objetivo describir los tipos de accesorios utilizados para la inmovilización y para la protección de pequeños animales sometidos a radioterapia. Para su desarrollo una búsqueda bibliográfica se realizó por los sitios de búsqueda como Google Scholar, Scielo, Bireme, PubMed, y consultas en la biblioteca libros campus de Botucatu UNESP. A pesar de las limitaciones de cada accesorio valorado este examen, fue posible identificar el uso de cada uno y cómo esto puede ser ventajoso para el tratamiento de los animales sometidos a radioterapia.
\end{abstract}

Palabras clave: la radioterapia veterinária, animales pequeños, accesorios de inmovilización.

\section{INTRODUÇÃO}

A radioterapia é uma modalidade médica bem estabelecida para o tratamento do câncer. $\mathrm{Na}$ área da medicina veterinária a radioterapia tem se apresentado como uma importante opção terapêutica na rotina da clínica de pequenos animais. No entanto, no Brasil, a aplicação da radioterapia veterinária é ainda pouco realizada, limitando-se apenas à pesquisas em poucos hospitais veterinários em algumas universidades do país $(1,2)$.

Os protocolos radioterápicos aplicados na veterinária variam em função de vários fatores, tais como: disponibilidade de equipamento e de fontes radioativas, fatores econômicos, preferências do cliente, manejo e logística do animal $(3,4)$. O estadiamento clínico da doença também é um fator preponderante para a escolha do plano de tratamento (5).

O sucesso da radioterapia depende de vários fatores, dentre os quais o uso de acessórios adequados para a proteção e imobilização dos pacientes. Estes dispositivos devem possuir características próprias que contribuem para o manuseio confortável do paciente e permitam a focalização do campo de radiação restrita ao volume de tratamento e não interfiram na dose de radiação liberada (6).

A maioria dos procedimentos radioterápicos em medicina veterinária requer a sedação ou mesmo a anestesia do animal. Este fato impõe o uso de acessórios de imobilização especialmente desenvolvidos para as diferentes espécies de animais tratados e conforme os respectivos procedimentos indicados (7). Por isso este trabalho teve como objetivo descrever os tipos de acessórios de imobilização e proteção utilizados na radioterapia de pequenos animais.

\section{MATERIAIS E MÉTODOS}

A pesquisa bibliográfica foi realizada por meio de sites de buscas como Google Acadêmico, Scielo, Bireme e PubMed, localizados com as palavras chaves: radioterapia veterinária, pequenos animais, acessórios de imobilização, além de consultas em livros da 
biblioteca do campus da UNESP de Botucatu, relacionados com o uso de radiações ionizantes em medicina veterinária.

Os periódicos, livros e publicações online encontrados foram analisados conjuntamente com um especialista na área e as publicações descritas em língua inglesa foram traduzidas manualmente e corrigidas por um revisor.

Para a imobilização dos pequenos animais durante o tratamento além da sedação e da anestesia, são utilizados alguns acessórios imobilizadores semelhantes aos usados na radioterapia humana e adaptados ao uso animal, tais como quadros ou molduras para cabeça, máscaras termoplásticas, blocos de mordida e colchões moldáveis (5).

As máscaras de material termoplástico, quando em temperatura ambiente são rígidas. Para a moldagem da região anatômica do paciente, a máscara é aquecida em água a uma temperatura de $70^{\circ} \mathrm{C}$ e em seguida é aplicada na região a ser moldada, após cerca de 5,0 minutos o material está novamente endurecido e assume a forma da região anatômica moldada e pronto para o seu uso (8).

O uso da máscara termoplástica tem demonstrado bastante aplicabilidade em pequenos animais, especialmente para o tratamento de tumores da cabeça e pescoço, quando comparados com outros acessórios imobilizadores da cabeça. No entanto, a efetividade da máscara termoplástica é ainda maior quando seu uso é em conjunto com outros acessórios de imobilização (9).

O preparo da máscara termoplástica segue a mesma praticidade dos serviços de radioterapia humana, no entanto, ao colocá-la em um paciente veterinário, em alguns casos é preciso modificá-la. Uma adaptação frequente é um corte circular na porção mais rostral do termoplástico, o qual permite uma abertura para a passagem do tubo endotraqueal. Os pacientes da veterinária são normalmente colocados em decúbito ventral para a montagem da máscara, enquanto os pacientes humanos são convencionalmente colocados em posição dorsal (10). A Figura 1 ilustra a utilização de uma máscara termoplástica adaptada ao uso de um cão, realçando a abertura para a passagem do tubo endotraqueal.

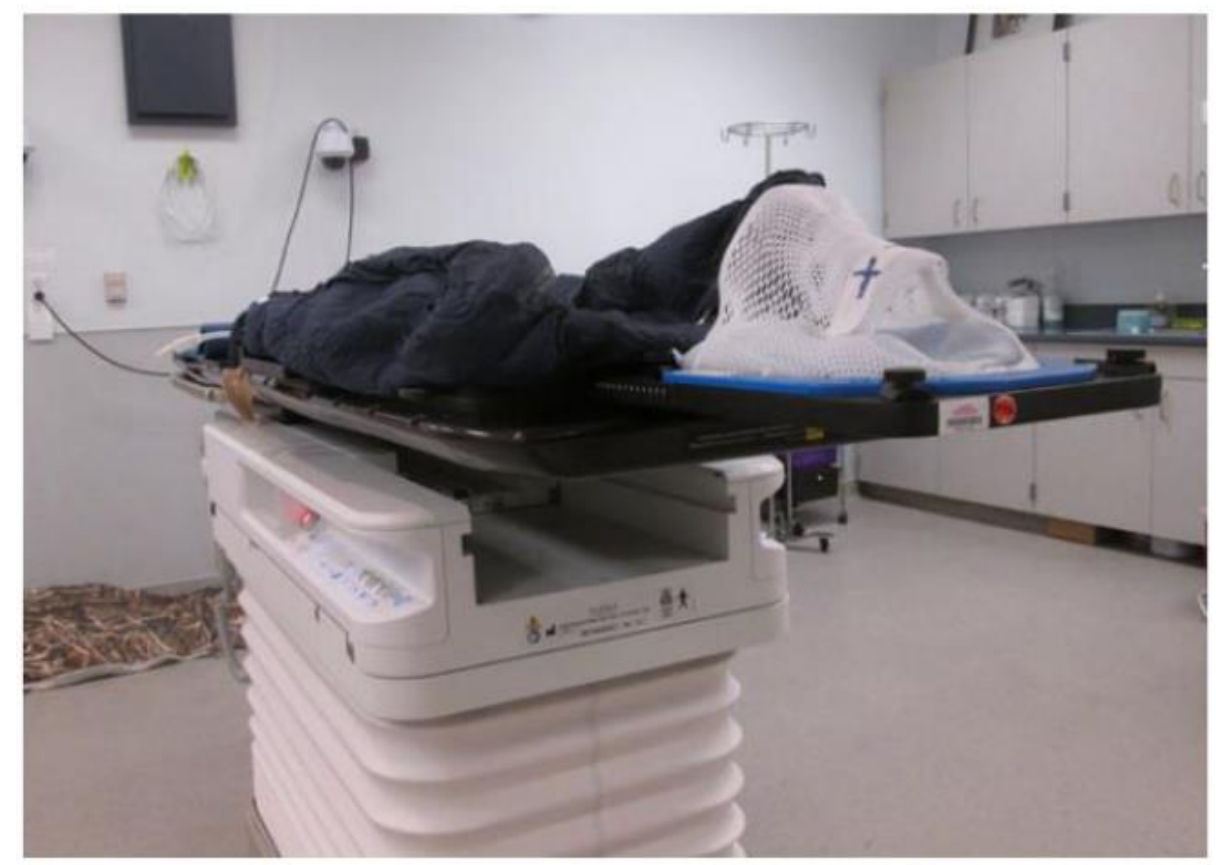

Figura 1. Máscara termoplástica adaptada ao uso veterinário na mesa de um equipamento de radioterapia (10). 
A máscara de imobilização deve ser utilizada durante todo o tratamento, no entanto, alguns fatores como o emagrecimento dos pacientes ou uma diminuição da lesão, implicará em reajuste do acessório ou na confecção de nova máscara, com subsequente planejamento do tratamento para se garantir a correta irradiação do volume alvo (11).

Alguns estudos $(12,13,14)$ apontam que o uso de máscaras termoplásticas intensifica a dose de entrada na pele do paciente.

Um acessório utilizado para a imobilização da região bucal do animal é o bloco de mordida confeccionado em resina de uso em prótese dentária (resina e catalisador). Durante o planejamento radioterápico, os dentes do paciente são pressionados na resina até o endurecimento do molde (15). Outros pesquisadores preferem blocos de mordida feitos em acrílico (16).

A Figura 2 ilustra a sequência de confecção do molde de mordida para imobilização do paciente veterinário até o momento do posicionamento para o procedimento de radioterapia (A a D) e o posicionamento de um cão um molde dentário de acrílico (E a G).
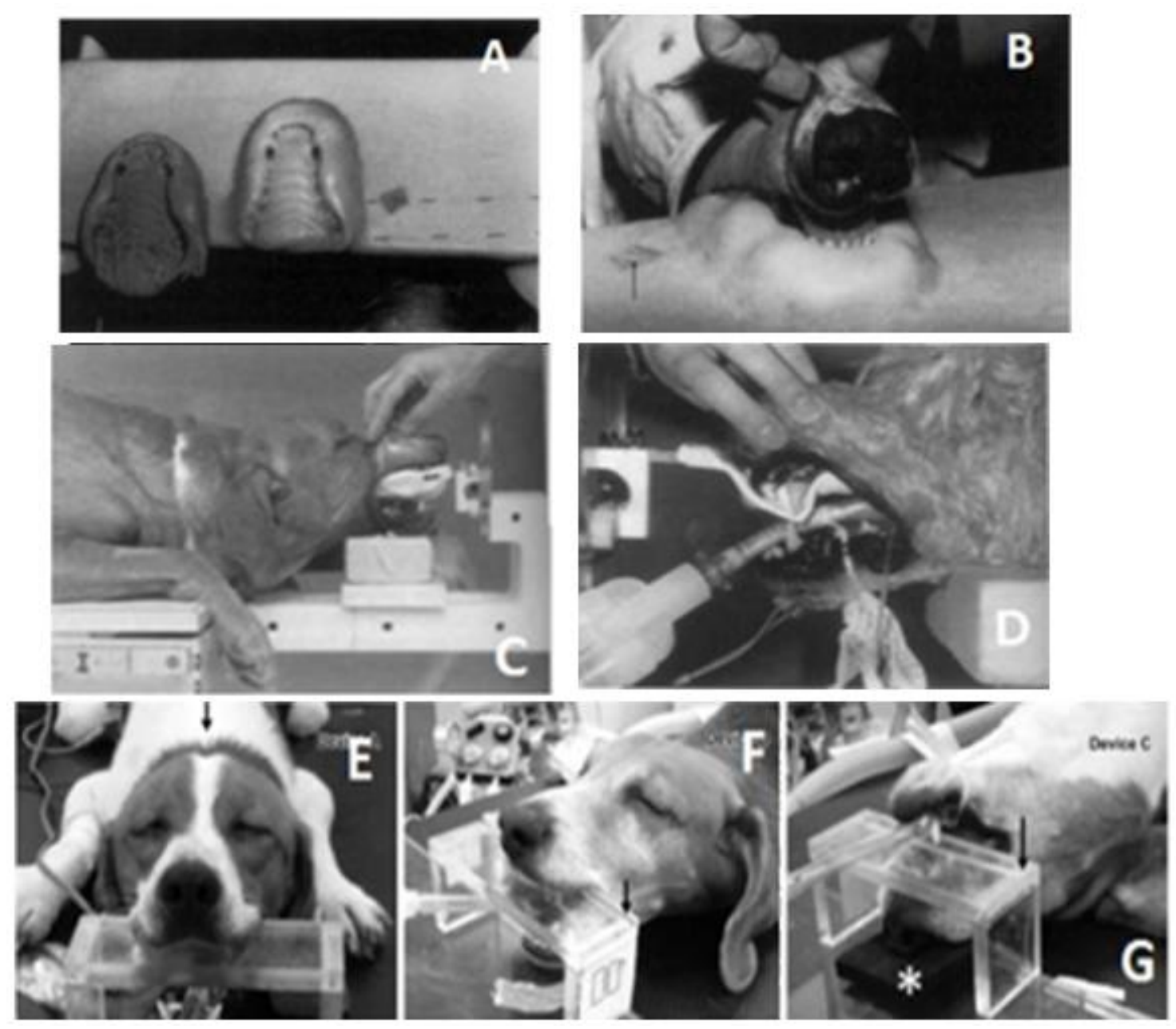

Figura 2. Posicionamento do animal durante processo de imobilização no acessório (A a D) e no molde dentário de acrílico (E a G) $(9,15,16)$.

Alguns autores indicam o uso de colchão inflável (vac-lok ${ }^{\mathrm{TM}}$ ) para imobilização do paciente (17). Este acessório substitui os convencionais, tais como: sacos de areia, toalhas, fitas de fixação, cunhas de espuma e outros. Uma desvantagem do método convencional em relação ao uso do colchão inflável é a falta de conformação ao contorno do paciente, o que pode provocar a variabilidade do posicionamento durante o tratamento. Além disto, o colchão inflável pode ser reutilizado em outro paciente. 
A Figura 3 ilustra o uso do colchão inflável no procedimento de aquisição das imagens tomográficas (A e B) e o posicionamento de um cão imobilizado para a realização da radioterapia $(\mathrm{C})$.

Outro acessório comumente usado é o compensador de tecido, que popularmente é denominado como "bolus", o qual é utilizado para preencher a região a ser tratada que apresenta falta de tecido, produzindo assim a superficialização da dose de radiação. A cera odontológica é um material comumente usado para confecção de bolus (18). A Figura 4 ilustra um cão com cicatriz cirúrgica após retirada do mastocitoma na região do membro (A) e em procedimento radioterápico com o uso de bolus $(\mathrm{B})$.

O filtro em cunha é um acessório utilizado para homogeneização da dose de radiação utilizado para compensar a falta de tecido no volume de tratamento. O filtro normalmente é confeccionado em material de alta densidade do tipo chumbo ou uma liga metálica com aço inoxidável, antimônio e cobre. As cunhas de formato triangular são posicionadas entre o feixe de radiação e o paciente, de modo que, o lado da cunha de maior espessura é colocado na direção de maior espessura de tecido, modificando a distribuição da dose de radiação, proporcionando maior planificação do perfil do campo de tratamento (8).
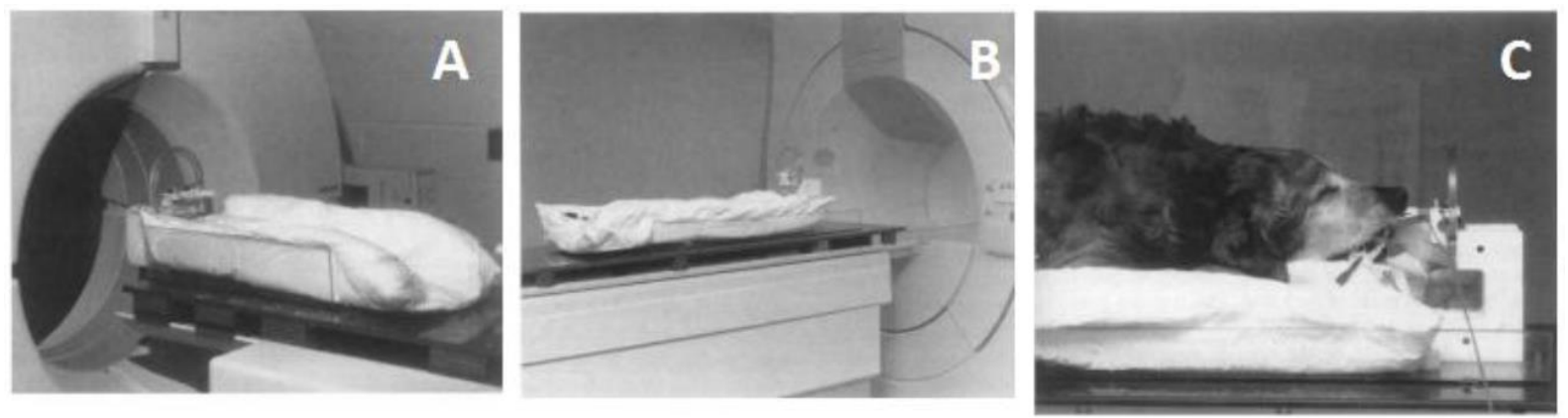

Figura 3. Colchão inflável (A e B) e seu uso em um cão (C) (9).
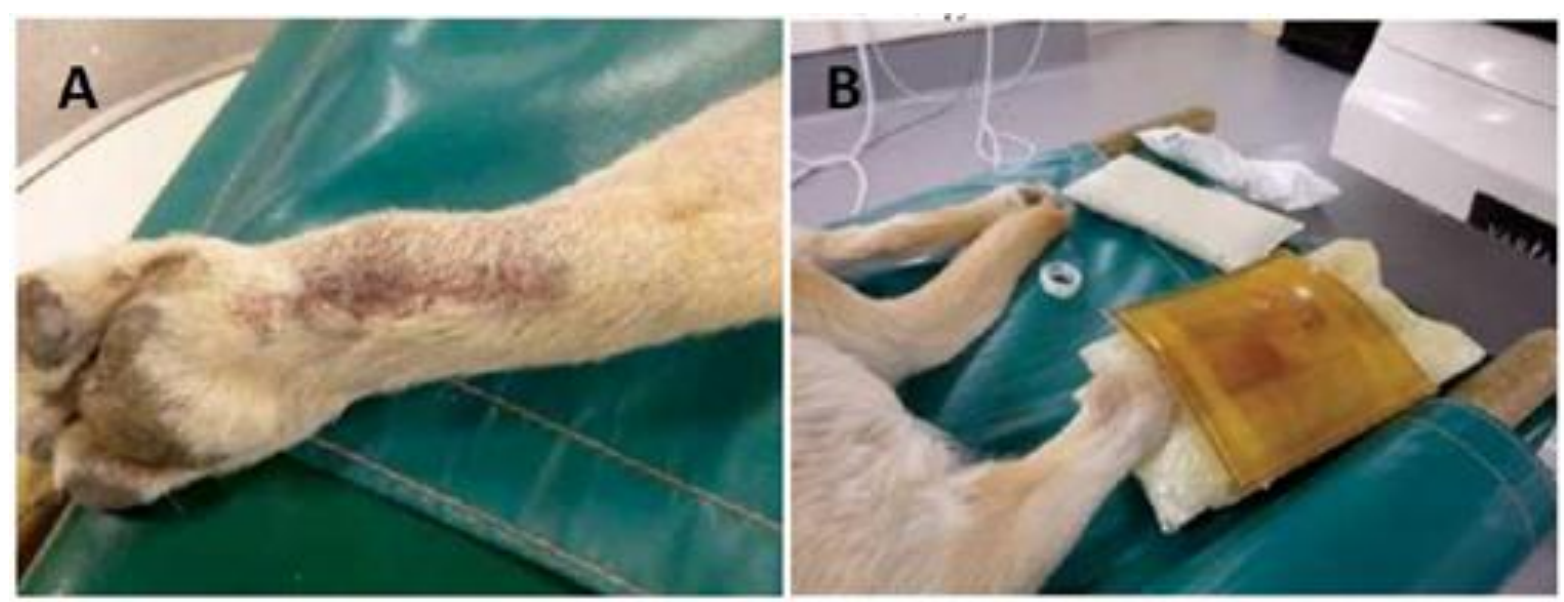

Figura 4. Cicatriz cirúrgica após a retirada do mastocitoma (A) e o uso de bolus na radioterapia do cão sobre a cicatriz (19).

Para os casos onde se necessitam proteger regiões anatômicas localizadas no interior do campo de radiação, são utilizados blocos de colimação que podem ser confeccionados em chumbo ou em liga metálica chamada de cerrobend (composta por chumbo, cadmio, antimônio e estanho. $(8,19)$.

A Figura 5 ilustra um cão em decúbito lateral frente ao cabeçote de um acelerador linear posicionado para radioterapia em adenocarcinoma de saco anal (A e C). A imagem realça o uso de bloco de colimação de chumbo para proteção dos tecidos normais (B). 
Técnicas modernas de radioterapia proporcionam melhor imobilização do volume alvo e garantem maior precisão na liberação da dose, propiciando maior controle local da doença $(20,21,22)$. Lesões próximas da região ocular podem utilizar lentes especiais de cerâmica e tungstênio para evitar a exposição de áreas sadias circunvizinhas, nestes casos superficiais, normalmente usam-se feixes de elétrons de alta energia (4 MeV a $15 \mathrm{MeV})(21,23)$.

A Tabela 1 ilustra um breve resumo a respeito dos acessórios de imobilização utilizados na radioterapia veterinária em pequenos animais, descrevendo o tipo de material, sua utilidade, e seus aspectos positivos e negativos no cotidiano.
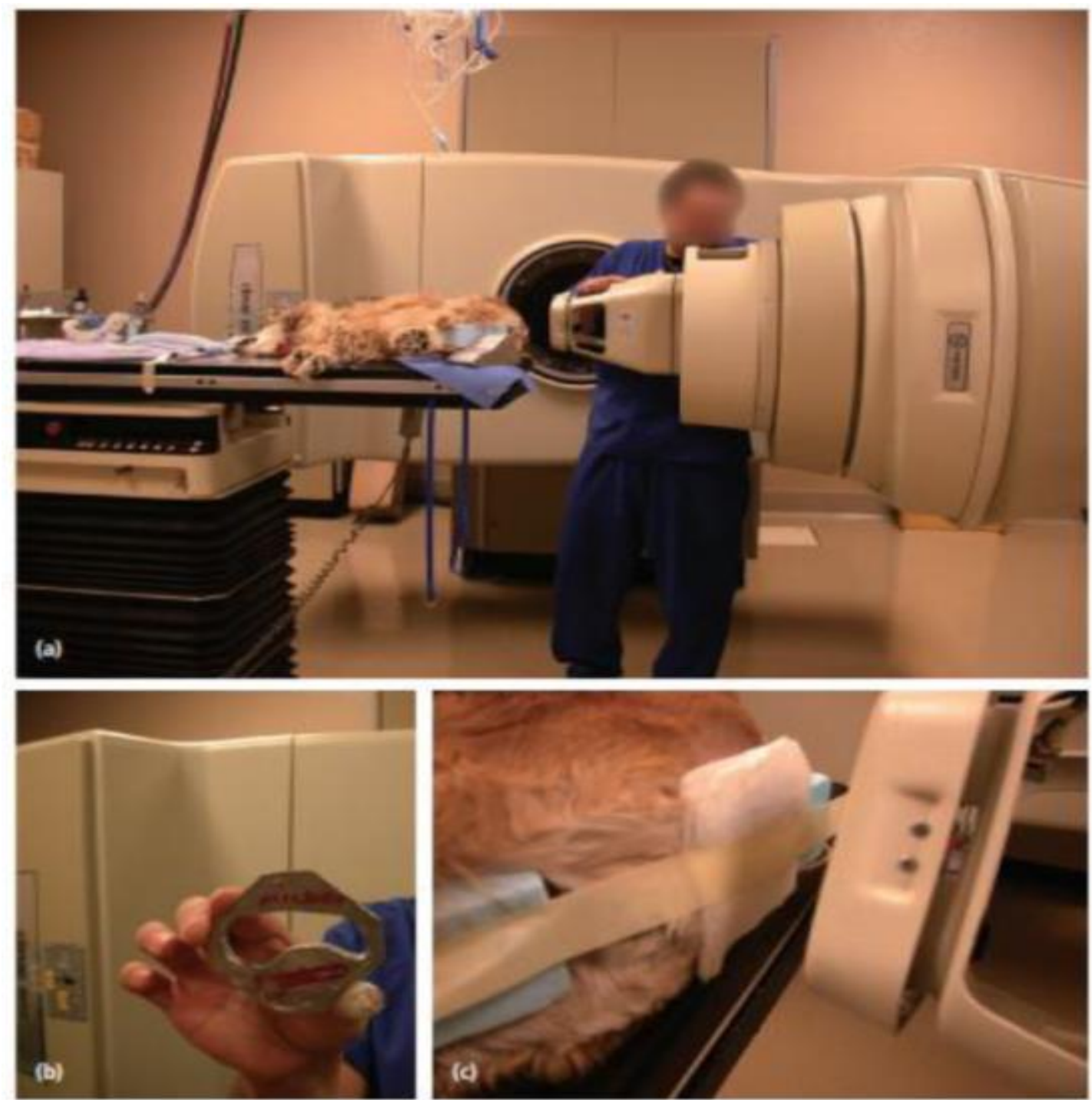

Figura 5. Cão submetido à radioterapia com acelerador linear em região anal (A e C) e a demonstração do bloco de colimação utilizado (19). 
Tabela 1. Resumo descritivo sobre os acessórios de proteção e de imobilização em pequenos animais.

\begin{tabular}{|c|c|c|c|c|}
\hline $\begin{array}{l}\text { Acessórios de } \\
\text { proteção e de } \\
\text { imobilização }\end{array}$ & Materiais & Utilidade & Vantagens & Desvantagens \\
\hline $\begin{array}{c}\text { Máscara } \\
\text { Termoplástica }\end{array}$ & $\begin{array}{l}\text { Material } \\
\text { termoplástico }\end{array}$ & $\begin{array}{l}\text { Imobilização da } \\
\text { região da cabeça e } \\
\text { pescoço }\end{array}$ & $\begin{array}{l}\text { Maior acessibilidade, maior } \\
\text { afinidade com outros } \\
\text { acessórios. } \\
\text { Ajuda a manter o paciente } \\
\text { corretamente posicionado e } \\
\text { também evita que o paciente } \\
\text { seja tatuado em alguma região } \\
\text { visível do corpo }\end{array}$ & $\begin{array}{l}\text { Pode ser desajustada } \\
\text { devido a fatores como } \\
\text { emagrecimento do } \\
\text { paciente ou diminuição da } \\
\text { lesão e potencializam o } \\
\text { aumento de dose à pele }\end{array}$ \\
\hline $\begin{array}{l}\text { Blocos de } \\
\text { Mordidas }\end{array}$ & $\begin{array}{c}\text { Materiais } \\
\text { odontológicos, } \\
\text { plástico ou acrílico }\end{array}$ & $\begin{array}{c}\text { Adaptados a } \\
\text { vários tratamentos }\end{array}$ & $\begin{array}{l}\text { Maior variedade de tamanho e } \\
\text { tipo de material, afinidade } \\
\text { com outros materiais e } \\
\text { acessórios. } \\
\text { Proporciona um } \\
\text { posicionamento que favorece } \\
\text { o tratamento sugerido }\end{array}$ & Pouco reaproveitável \\
\hline $\begin{array}{l}\text { Colchão } \\
\text { Inflável } \\
\text { (Vac-Lok) }\end{array}$ & Colchão de isopor & $\begin{array}{c}\text { Adaptados a } \\
\text { vários tratamentos }\end{array}$ & $\begin{array}{l}\text { Acessório reutilizável, melhor } \\
\text { imobilização do que os } \\
\text { procedimentos convencionais } \\
\text { (sacos de areia, toalhas, fita, } \\
\text { cunhas de espuma) e } \\
\text { afinidade com outros } \\
\text { acessórios }\end{array}$ & $\begin{array}{l}\text { A falta de conformação } \\
\text { no contorno do paciente, } \\
\text { comprometendo o } \\
\text { tratamento, pode } \\
\text { apresentar problemas no } \\
\text { posicionamento de } \\
\text { membros pequenos }\end{array}$ \\
\hline Bólus & $\begin{array}{c}\text { Uma variedade de } \\
\text { materiais, sendo cera } \\
\text { odontológica a mais } \\
\text { comum }\end{array}$ & $\begin{array}{c}\text { Usados em } \\
\text { tumores que } \\
\text { envolvem a pele } \\
\text { ou próximo a ela }\end{array}$ & $\begin{array}{c}\text { Proporciona a melhor } \\
\text { distribuição de dose nos } \\
\text { tecidos e superficializa a dose } \\
\text { de radiação }\end{array}$ & Pouco reaproveitável \\
\hline $\begin{array}{l}\text { Blocos de } \\
\text { Colimação }\end{array}$ & $\begin{array}{l}\text { Chumbo ou } \\
\text { cerrobend }\end{array}$ & $\begin{array}{c}\text { Adaptados a } \\
\text { vários tratamentos }\end{array}$ & $\begin{array}{l}\text { Pode ser aproveitado, é } \\
\text { facilmente personalizado com } \\
\text { o formato do paciente }\end{array}$ & Colimação limitada \\
\hline Balão Retal & $\begin{array}{l}\text { Plástico inflável com } \\
\text { ar }\end{array}$ & $\begin{array}{l}\text { Usado no } \\
\text { tratamento de } \\
\text { próstata }\end{array}$ & $\begin{array}{l}\text { Minimiza o movimento da } \\
\text { próstata durante o tratamento }\end{array}$ & Pouco reaproveitável \\
\hline $\begin{array}{c}\text { Lentes } \\
\text { Protetoras }\end{array}$ & $\begin{array}{l}\text { Lente coberta com } \\
\text { cerâmica em contato } \\
\text { com um bloco de } \\
\text { tungstênio }\end{array}$ & $\begin{array}{c}\text { Usadas no } \\
\text { tratamento de } \\
\text { tumores próximos } \\
\text { aos olhos }\end{array}$ & $\begin{array}{l}\text { Diminuição significativa da } \\
\text { dose em torno do olho }\end{array}$ & $\begin{array}{l}\text { Pouco reaproveitavél e } \\
\text { pouca utilização (apenas } \\
\text { em tratamentos próximo } \\
\text { ao olho com feixes de } \\
\text { elétrons) }\end{array}$ \\
\hline
\end{tabular}

\section{CONSIDERAÇÕES FINAIS}

Ao contrário dos acessórios básicos usados em um serviço de radiologia veterinária como sacos de areia, toalhas, fitas, cunhas de espuma, entre outros, uma ampla variedade de acessórios de proteção e imobilização podem ser utilizados em um serviço de radioterapia veterinária de pequenos animais.

Cada acessório tem suas vantagens e suas limitações no cotidiano. No entanto é possível observar que apenas alguns deles podem ser adaptados a vários tipos de tratamentos e outros para alguns mais específicos conforme cada enfermidade. Todos os acessórios descritos procuram fornecer o melhor conforto para o paciente, além da imobilização e sua proteção. Nem todos os acessórios podem ser reaproveitáveis para utilização futura em outros pacientes.

Alguns acessórios levam tempo para serem preparados, o que para alguns serviços com grande rotina, podem atrasar o seu andamento, necessitando de mais profissionais capacitados para realizarem esses procedimentos evitando-se riscos. A demora na preparação dos 
materiais pode ser compensada com a eficácia de cada um deles e em alguns casos, com a sua reutilização em outros pacientes.

Apesar das limitações de cada acessório, é possível identificar a sua utilização em conjunto e podendo ser vantajoso para o tratamento. Não é possível dizer qual é a melhor combinação, ou qual é o melhor acessório para imobilizar o paciente. É possível recomendar o melhor acessório ou a melhor combinação para cada tipo tratamento.

\section{REFERÊNCIAS}

1. Fernandes MAR, Andrade AL, Luvizoto MCR, Pierô JR, Ciarlini LDRP. Radioterapia em medicina veterinária princípios e perspectivas. Rev Bras Fis Med. 2010;4(2):11-4.

2. Morris J, Dobson J. Oncologia de pequenos animais. São Paulo: Roca; 2007. p.55-6.

3. Boston S, Henderson RA. Role of surgery in multimodal cancer therapy for small animals. Vet Clin North Am Small Anim Pract. 2014;44(5):855-70.

4. Burk RL, King GK. The veterinay clinics of north America: small animal practice. radiation oncology. Philadelphia: WB Saunders Company; 1997.

5. Schwarz T, Saunders J. Veterinary computed tomography. Ames: John Wiley and Sons; 2011.

6. Andrade LA, Fernandes MAR. Braquiterapia em medicina veterinária. In: Jericó MM, Neto AJP, Kogika MM. Tratado de medicina interna de cães e gatos. São Paulo: Roca; 2014. v.2, p.560- 83.

7. Lage MFAA, Mercicano AD, Zuliani GC. Radioterapia In: Nobrega AL. Tecnologia radiológica e diagnóstico por imagem. 4a ed. São Caetano do Sul: Difusão; 2012. p.24764.

8. Pereira AJ, Daniliauskas M, Lopes RMG, Haddad GP, Cruz JC, Martins HL. Manual para técnicos em radioterapia. Rio de Janeiro: Instituto Nacional de Câncer; 2000.

9. Kippenes H, Gavin PR, Sande RD, Rogers D, Sweet V. Comparison of the accuracy of positioning devices for radiation therapy of canine and feline head tumors. Vet Radiol Ultrasound. 2000;41(4):371-6.

10. Hansen KS, Théon AP, Doeteroch S, Kent MS. Validation of indexed radiotherapy head positioning device for use in dogs and cats. Vet Radiol Ultrasound. 2015;56(4):448-55.

11. Caetano MAA. Estudo de três sistemas de imobilização utilizados em radioterapia perspetiva atual e futura [dissertação]. Lisboa: Escola Superior de Tecnologia da Saúde de Lisboa; 2014.

12. Levitt SH, Purdy JA, Perez CA, Poortmans P. Technical basis of radiation therapy pratical clinical applications. Berlin: Spinger; 2012. 
13. Zoltán Varga KH, Tamás Mezo AN, Thurzó L, Kahán Z. Individual positioning: a comparative study of adjuvant breast radiotherapy in the prone versus supine position. Int J Radiat Oncol Biol Phys. 2009;75(1): 94-100.

14. Bentel GC, Marks LB, Sherouse GW, Spencer DP, Anscher MS. The effectiveness of immobilization during prostate irradiation. Int $J$ Radiat Oncol Biol Phys. 1995;31(1):143-8.

15. Bley CR, Blattmann H, Ross M, Sumova A, Kaser-Hotz B. Assessment of a radiotherapy patient immobilization device using single plane port radiographs and a remote computed tomography scanner. Vet Radiol Ultrasound. 2003;44(4):470-5.

16. Maruo T, Nakamura S, Fukuyama Y, Kawarai S. Validation of new bite block-type headimmobilization devices por radiotherapy in dogs. Vet Radiol Ultrasound. 2013;54(6):674-9.

17. Green EM, Forrest LJ, Adams WM. A vacuum-formable mattress for veterinary radiotherapy positioning: comparison with conventional methods. Vet Radiol Ultrasound. 2003;44(4):476-9.

18. Salvajoli JV, Souhami L, Faria SL. Radioterapia em oncologia. São Paulo: Atheneu; 2013.

19. Kudning ST, Séguin B. Veterinary surgical oncology. Ames: Wiley Blackwell; 2012.

20. Withrow SJ, Vail DM, Page R. Withrow and MacEwen's small animal clinical oncology. 5th ed. St. Louis: Elsevier Saunders; 2013.

21. Marcinowska A, Dobson J. Radiotherapy and tumours in veterinary practice: part one [Internet]. Veterinary Times; 2015 [cited 2017 Jun 25]. Avaialble from: https://www.vettimes.co.uk/article/radiotherapy-and-tumours-in-veterinary-practice-partone/

22. Harmon JJr, Yoshikama H, Custis J, Larue S. Evaluation of canine prostate intrafractional motion using serial cone beam computed tomography imaging. Vet Radiol Ultrasound. 2013;54(1):93-8.

23. Larue SM, Custis JT. Advances in veterinary radiation therapy targeting tumors and improving patient comfort. Vet Clin North Am Small Anim Pract. 2014;44(5):909-23.

Recebido em: 02/08/2016 Aceito em: 03/07/2017 